\title{
A State Space Approach for the Eigenvalue Problem of Marine
}

\section{Risers}

\author{
Feras K. Alfosail $^{1} \quad$ Ali H. Nayfeh ${ }^{2,3} \quad$ Mohammad I. Younis ${ }^{1,4^{*}}$
}

${ }^{1}$ Physical Sciences and Engineering Division, King Abdullah University of Science and Technology, Thuwal 23955-9600, Saudi Arabia.

${ }^{2}$ Department of Engineering Science and Mechanics, MC 0219, Virginia Polytechnic Institute and State University, Blacksburg, VA 24061, U.S.A.

${ }^{3}$ University of Jordan, Amman, Jordan.

${ }^{4}$ Department of Mechanical Engineering, State University of New York, Binghamton, NY 13902, USA

\begin{abstract}
A numerical state-space approach is proposed to examine the natural frequencies and critical buckling limits of marine risers. A large axial tension in the riser model causes numerical limitations. These limitations are overcome by using the modified Gram-Schmidt orthonormalization process as an intermediate step during the numerical integration process with the fourth-order Runge-Kutta scheme. The obtained results are validated against those obtained with other numerical methods, such as the finite-element, Galerkin, and power-series methods, and are found to be in good agreement. The state-space approach is shown to be computationally more efficient than the other methods. Also, we investigate the effect of a high applied tension, a high apparent weight, and higher-order modes on the accuracy of the numerical scheme. We


demonstrate that, by applying the orthonormalization process, the stability and convergence of the approach are significantly improved.

\section{INTRODUCTION}

The study of the linear dynamics of marine risers is a major topic in fluid-structure interactions. The main objective of the linear analysis is to understand how the eigenvalues (frequencies) of the riser depend on its parameters, such as the applied tension and apparent weight. The linear analysis is the first step in studying the nonlinear dynamics of risers, such as vortex-induced vibrations (VIV) [1-3], and its dependence on the geometric features of the risers [4-6]. The outcome of such studies provides an understanding of how a marine riser behaves and hence prevents its failure from fatigue [7].

The interest in riser dynamics began when Huang and Dareing [8] employed a powerseries (Forbenius) method to find the buckling limits and natural frequencies of marine drilling risers with linearly varying axial tension. In a subsequent work [9], the results from the powerseries were compared with those of a beam with a constant axial tension equal to the average tension of the riser. Paidoussis [10] found that 50 terms in the power series are required to determine the buckling limits to three significant digits. Kirk et al. [11] used the Rayleigh-Ritz method utilizing the mode shapes of a straight beam. They achieved good agreement with the results of Huang and Dareing [8] when the applied tension is greater than the apparent weight. Paidoussis [12] and Holmes [13] used, respectively, the Galerkin-Ritz method and Galerkin averaging to investigate the buckling aspects of a riser pipe due to an internal flow. Convergence of the numerical results in these studies required a minimum of five to six beam mode shapes. 
An alternative approach for studying the linear dynamics of risers is perturbation methods. Kim [12] used the Wentzel-Kramers-Brillouin (WKB) method to obtain an integral equation governing the mode shapes and natural frequencies. The results of the WKB are in good agreement with the power-series results. The agreement improves for higher-order modes of tension dominated risers. However, in general, the WKB overestimates the natural frequencies for partially tensioned risers when compared to the upper bounds of Laird and Fauconneau [13]. In addition, the WKB is valid when the coefficients in the equation are slowly varying. Several authors [14-16] used a straightforward perturbation method to find approximations for the eigenvalue problem of tension dominated risers by neglecting the effects of bending, thereby reducing the governing equation into a Bessel equation. However, the resulting solution is inaccurate because it does not satisfy all of the boundary conditions. This motivated Mazzilli et al. [17] to obtain a closed-from solution considering bending and tension aspects of the riser using perturbation methods. Lately, Clementi et al. [4] used the method of multiple scales [18] to determine a closed-form expression for the natural frequencies of risers taking into account its nonlinear effects..

On the other hand, approximate techniques have been proposed to study the linear dynamics of risers. In the case of tension dominant risers, the bending term is neglected, thereby reducing the governing equation into a Bessel equation [19]. Senjanović, et al. [21], Sparks [22], and Graves and Dareing [23] used a segmentation method to account for bending effects. Chen et al. [20] used the differential quadrature method to transform the differential equation into a recursive equation and calculate the natural frequencies. The rate of convergence increases when the average tension is used. Chen et al. [21] used a variational iteration technique with the assumed mode shape obtained by virtue of a Lagrange multiplier. Then, the frequencies and mode shapes 
are obtained by applying the boundary conditions. The convergence rate of the variational iteration technique is faster than that of the differential quadrature method.

Numerical techniques, such the finite difference or the finite element, present a computational challenge for tension dominated risers due to the large number of elements required to resolve the regions near the boundaries. Huan et al. [22] used the von Neumann stability to demonstrate that the finite difference scheme is unconditionally stable only if the variable tension is replaced by a constant value. Chen et al. [23, 24] combined the dynamic stiffness method and the WKB to improve the finite element scheme. The results show that the number of elements is reduced significantly when compared to a standard finite element method. Although the results obtained with this method agree well with previously published work, it imposes a limitation on the parameters used in the analysis.

Due to the loss of generality of the modified problem in the aforementioned numerical schemes, we calculate the natural frequencies of marine risers using a numerical state-space approach in which the governing boundary-value problem is transformed into an initial value problem. Then, the four-dimensional state-space system is integrated using the fourth-order Runge-Kutta method and the modified Gram-Schmidt orthonormalization method is used to keep the obtained four solutions linearly independent. The method was introduced and detailed by Scott and Whats [25] and has been used previously in free vibration studies of other structures, such as plates [26]. However, it has never been applied to vertical marine risers. Therefore, we apply this method to the linear dynamics of vertical marine risers. 




Figure 1. A schematic of a vertical riser extending from the surface level to the seabed.

We consider the marine riser in Fig 1. The differential equation governing the motion of the vertical riser is written as [27]

$$
\begin{aligned}
& m \frac{\partial^{2} \hat{y}}{\partial t^{2}}+E I \frac{\partial^{4} \hat{y}}{\partial \hat{x}^{4}}+2 m_{f} U_{i} \frac{\partial^{2} \hat{y}}{\partial \hat{x} \partial t}+\left(m_{f} U i^{2}-\left(T_{e}-W_{e}(L-\hat{x})\right)-\frac{E A_{p}}{2 L} \int_{0}^{L}\left[\left(\frac{\partial \hat{y}}{\partial \hat{x}}\right)^{2}\right] d \hat{x}\right)\left(\frac{\partial^{2} \hat{y}}{\partial \hat{x}^{2}}\right) \\
& -W_{e}\left(\frac{\partial \hat{y}}{\partial \hat{x}}\right)+c \frac{\partial \hat{y}}{\partial t}=F
\end{aligned}
$$

where $m$ is the total mass per unit length expressed as $m=m_{f}+m_{p}+m_{a}, m_{f}$ is the mass of the internal fluid per unit length, $m_{p}$ is the mass of the riser structure, $m_{a}$ is the fluid added mass, $E I$ is the flexural rigidity, $U_{i}$ is the internal fluid velocity, $T_{e}$ is the applied tension, $L$ is the length of the riser, $A_{p}$ is the cross-section area of the riser, $W_{e}$ is the apparent weight of the riser per unit length, $c$ is the structural damping of the riser per unit length, and $F$ is the external force 
acting on the riser per unit length. For simplification, we introduce the following dimensionless variables:

$y_{d}=\frac{\hat{y}}{D} ; \quad x=\frac{\hat{x}}{L} ; \quad t=\sqrt{\frac{E I}{m L^{4}}} \hat{t}$

where the superscript “^” denotes dimensional variables and the variables without "^» are dimensionless. Substituting Eq. (2) into Eq. (1), we obtain

$$
\begin{aligned}
& \frac{\partial^{2} y_{d}}{\partial t^{2}}+\frac{\partial^{4} y_{d}}{\partial x^{4}}+2 \sqrt{\beta} \bar{v} \frac{\partial^{2} y_{d}}{\partial x \partial t}+\left(\bar{v}^{2}-(\bar{T}-\bar{\sigma}(1-x))-\bar{\eta}\left(\int_{0}^{L}\left[\left(\frac{\partial y_{d}}{\partial x}\right)^{2}\right] d x\right)\right)\left(\frac{\partial^{2} y_{d}}{\partial x^{2}}\right)-\bar{\sigma}\left(\frac{\partial y_{d}}{\partial x}\right)+\bar{c} \frac{\partial y_{d}}{\partial t}=\bar{F} \\
& \beta=\frac{m_{f}}{m} \quad \bar{v}=\sqrt{\frac{m_{f}}{E I}} L U_{i} \quad \bar{\sigma}=\frac{W_{e} L^{3}}{E I} \quad \bar{\eta}=\frac{A_{p} D^{2}}{2 I} \quad \bar{c}=\frac{c L^{2}}{\sqrt{m E I}} \\
& \bar{F}=\frac{F L^{4}}{E I D} \quad \bar{T}=\frac{T_{e} L^{2}}{E I}
\end{aligned}
$$

To determine the mode shapes and natural frequencies, we linearize Eq. (3), drop the forcing and damping terms, and obtain

$$
\frac{\partial^{2} y_{d}}{\partial t^{2}}+\frac{\partial^{4} y_{d}}{\partial x^{4}}+2 \sqrt{\beta} \bar{v} \frac{\partial^{2} y_{d}}{\partial x \partial t}+\left(\bar{v}^{2}-(\bar{T}-\bar{\sigma}(1-x))\right)\left(\frac{\partial^{2} y_{d}}{\partial x^{2}}\right)-\bar{\sigma}\left(\frac{\partial y_{d}}{\partial x}\right)=0
$$

Next, we seek solutions of Eq. (5) in the form

$$
y_{d}(x, t)=\phi(x) e^{i \omega t}
$$

Substituting Eq. (6) into Eq. (5) and keeping the real part, we obtain

$$
\frac{d^{4} \phi}{d x^{4}}-(\alpha+\bar{\sigma} x)\left(\frac{d^{2} \phi}{d x^{2}}\right)-\bar{\sigma}\left(\frac{d \phi}{d x}\right)-\omega^{2} \phi(x)=0
$$


where $\alpha=\bar{T}-\bar{v}^{2}-\bar{\sigma}$. The imaginary part of the velocity $i 2 \omega \sqrt{\beta} \bar{v} \frac{d \phi}{d x}$ produces a gyroscopic effect which does not affect the free vibration of the riser structure [28]. Hence, the assumption to examine the real part of Eq. (5) is valid for the eigenvalue problem herein. In what follows, we consider the following three sets of boundary conditions: fixed-fixed, fixed-hinged, and hingedhinged boundary conditions.

We introduce the state variables

$z_{1}=\phi(x), z_{2}=\frac{d \phi}{d x}, z_{3}=\frac{d^{2} \phi}{d x^{2}}, z_{4}=\frac{d^{3} \phi}{d x^{3}}$

and transform Eq. (7) into the following system of four first-order ordinary differential equations:

$$
\begin{aligned}
& z_{1}{ }^{\prime}(x)=z_{2}(x) \\
& z_{2}{ }^{\prime}(x)=z_{3}(x) \\
& z_{3}{ }^{\prime}(x)=z_{4}(x) \\
& z_{4}{ }^{\prime}(x)=\omega^{2} z_{1}(x)+\bar{\sigma} z_{2}(x)+[\alpha+\bar{\sigma} x] z_{3}(x)
\end{aligned}
$$

Equations (9a) can be expressed in the state-space form

$\left[\mathrm{z}^{\prime}(x)\right]=[A(x)][z(x)]$

where

$$
\mathrm{Z}=\left[z_{1}(x) z_{2}(x) z_{3}(x) z_{4}(x)\right]^{T}
$$




$$
A=\left(\begin{array}{cccc}
0 & 1 & 0 & 0 \\
0 & 0 & 1 & 0 \\
0 & 0 & 0 & 1 \\
\omega^{2} & \bar{\sigma} & \alpha+\bar{\sigma} x & 0
\end{array}\right)
$$

The boundary conditions are expressed using the states defined in Eq. (8) as follows:

Fixed-Fixed:

$z_{1}(0)=0, z_{2}(0)=0, z_{1}(1)=0, z_{2}(1)=0$

Fixed-hinged:

$$
z_{1}(0)=0, z_{2}(0)=0, z_{1}(1)=0, z_{3}(1)=0
$$

Hinged-hinged:

$$
z_{1}(0)=0, z_{3}(0)=0, z_{1}(1)=0, z_{3}(1)=0
$$

The boundary conditions (11a), (11b), and (11c) can be expressed in matrix form as $R_{1} \mathrm{z}=0$ at $x=0$ and $R_{2} \mathrm{z}=0$ at $x=1$

where $R_{1}$ and $R_{2}$ are defined as

$$
\begin{aligned}
& R_{1}=\left(\begin{array}{llll}
1 & 0 & 0 & 0 \\
0 & 1 & 0 & 0
\end{array}\right) \text { and } R_{2}=\left(\begin{array}{llll}
1 & 0 & 0 & 0 \\
0 & 1 & 0 & 0
\end{array}\right) \text { for fixed-fixed boundary conditions } \\
& R_{1}=\left(\begin{array}{llll}
1 & 0 & 0 & 0 \\
0 & 1 & 0 & 0
\end{array}\right) \text { and } R_{2}=\left(\begin{array}{llll}
1 & 0 & 0 & 0 \\
0 & 0 & 1 & 0
\end{array}\right) \text { for fixed-hinged boundary conditions } \\
& R_{1}=\left(\begin{array}{llll}
1 & 0 & 0 & 0 \\
0 & 0 & 1 & 0
\end{array}\right) \text { and } R_{2}=\left(\begin{array}{llll}
1 & 0 & 0 & 0 \\
0 & 0 & 1 & 0
\end{array}\right) \text { for hinged-hinged boundary conditions }
\end{aligned}
$$


The general solution to Eq. (9b) is written as

$[z(x)]=\left[\phi\left(x, x_{0}\right)\right]\left[c_{0}\right]$

where $\left[\phi\left(x, x_{0}\right)\right]$ is the fundamental matrix obtained using a fourth-order Runge Kutta scheme and $x_{0}$ is the initial starting point of the integration scheme i.e. $x_{0}=0$. Convergence of the integration scheme is based on the norm of the error estimate, which determines the number of integration steps required for each initial vector. The integration process is started by defining the following four linearly independent initial vectors:

$\left[\phi\left(x_{0}, x_{0}\right)\right]=[I]$

Then, it follows from $R_{1} \mathrm{z}=0$ that

$\mathrm{z}(0)=c_{1} \mathrm{z}^{(1)}(0)+c_{2} \mathrm{z}^{(2)}(0)$

where $c_{1}$ and $c_{2}$ are unknown constants that linearly combine the vectors. Upon integrating the system of equations, one finds that the rapidly growing terms dominate the slowly varying ones, resulting in the loss of linear independence of the computed vectors [29]. From a numerical precision point of view, the machine will round off the numbers such that the smaller digits are neglected, thereby resulting in loss of precision when computing the eigenvalues [30]. To overcome this problem, we use the modified Gram-Schmidt orthonormalization procedure. To this end, we divide the length of the riser into equal segments such that the length of each segment is written as

$h=x_{k+1}-x_{k}$ 
Then, we integrate numerically the system of Eq. (9) and obtain for each segment the local matrix solution

$\left[\phi\left(x_{k+1}, x_{k}\right)\right]=\left\{\begin{array}{c}{\left[\phi\left(x_{1}, x_{0}\right)\right] 0 \leq x<x_{1}} \\ {\left[\phi\left(x_{2}, x_{1}\right)\right] x_{1} \leq x<x_{2}} \\ \vdots\end{array}\right.$

To ensure linear independence of the output vectors, we introduce the modified Gram-Schmidt orthonormalization procedure at the end of each step. We use the resulting vectors as initial conditions for the next interval. The process is continued until two linearly independent vectors are found at $x=1$, and hence the solution is expressed as

$\mathrm{z}(1)=\tilde{c}_{1} \tilde{\mathbf{z}}^{(1)}(1)+\tilde{c}_{2} \tilde{\mathrm{z}}^{(2)}(1)$

where $\tilde{c}_{1}$ and $\tilde{c}_{2}$ are unknown constant. Substituting Eq. (18) into $R_{2} \mathrm{z}=0$ yields

$R_{2} \tilde{Z} \tilde{c}=0$

where $\tilde{Z}$ is a $4 \times 2$ matrix whose columns are the vectors obtained from Eq. (19) and $\tilde{c}$ is a constant column vector. For a nontrivial solution,

$\operatorname{det}\left(R_{2} \tilde{Z}\right)=0$

The determinant defined by Eq. (20) determines the natural frequencies of the riser.

\section{NUMERICAL RESULTS}

1. Validation of the Results

To validate the numerical procedure, we compare our results with the results obtained by Dareing and Huang [9] for the riser properties defined in Table 1. 
Table 1. Riser properties used in the calculations.

\begin{tabular}{|c|c|}
\hline Riser length, $L$ & $500 \mathrm{ft}$ \\
\hline Riser weight per unit length, $W_{-} e$ & $214 \mathrm{lb} / \mathrm{ft}$ \\
\hline Interior cross-section area of the riser, $A_{i}$ & $2.99 f t^{2}$ \\
\hline Exterior cross-section area of the riser, $A_{e}$ & $3.14 f t^{2}$ \\
\hline Young's Modulus, $E$ & $30 \times 10^{6} l b / \mathrm{in}^{2}$ \\
\hline Moment of inertia, $I$ & $3136.9 \mathrm{in}^{4}$ \\
\hline Density of sea water, $\rho_{w}$ & $64.8 \mathrm{lb} / \mathrm{ft}^{3}$ \\
\hline Density of internal fluid, $\rho_{m}$ & $85 \mathrm{lb} / \mathrm{ft}^{3}$ \\
\hline Tension in the riser bottom, $T_{b}$ & 286 kips \\
\hline Mass of the riser per unit length, $m$ & 20.8 slugs/ft \\
\hline
\end{tabular}

We use the following parameters:

$$
\left\{\begin{array}{l}
\bar{\sigma}=\frac{144 W_{e} L^{3}}{E I} \\
\alpha=\frac{144\left(\bar{T}-\bar{v}^{2}-\bar{\sigma}\right) L^{2}}{E I} \\
\lambda^{4}=\frac{144 m \omega^{2} L^{4}}{E I}
\end{array}\right\}
$$

Hence, the parameters in equation (7) are $\bar{\sigma}=50.62$ and $\alpha=99.71$. To compute the roots of Eq. (20), we use a tolerance of $10^{-8}$. Validation of the results is presented in Table 2 . We compare the results obtained with the state-space method with those obtained with the Galerkin discretization using six straight beam mode shapes, the finite element using the COMSOL software [31], the power-series method [9], and the Chebyshev tau method [32]. A detailed analysis of the convergence of each method is given in Appendix A. 
Table 2. Lowest five natural frequencies of the riser.

\begin{tabular}{ccccccc}
\hline Mode & $\begin{array}{c}\text { Galerkin } \\
\text { Expansion }\end{array}$ & $\begin{array}{c}\text { Finite } \\
\text { Element }\end{array}$ & $\begin{array}{c}\text { Chebyshev } \\
\text { tau method }\end{array}$ & $\begin{array}{c}\text { Power } \\
\text { Series }\end{array}$ & $\begin{array}{c}\text { State Space } \\
\text { Current }\end{array}$ & $\begin{array}{c}\text { Absolute } \\
\text { relative deviation \% }\end{array}$ \\
\hline 1 & 0.81504 & 0.81507 & 0.81503 & 0.81498 & 0.81503 & 0.00613 \\
2 & 1.80369 & 1.80366 & 1.80369 & 1.80362 & 1.80369 & 0.00388 \\
3 & 3.08777 & 3.08785 & 3.08777 & 3.08762 & 3.08776 & 0.00453 \\
4 & 4.73754 & 4.73675 & 4.73754 & 4.73749 & 4.73754 & 0.00105 \\
5 & 6.78935 & 6.78748 & 6.78935 & 6.78901 & 6.78935 & 0.00500 \\
\hline
\end{tabular}

The results, in general, are in good agreement and the relative percentage errors of the lowest five modes are $\mathrm{O}\left(10^{-3}\right)$ when the results of the state-space method are compared to the power-series solution. The modified Gram-Schmidt orthonormalization procedure was only introduced in the final step. Table 3 shows a comparison of the time taken to discretize the governing equation and compute the frequency of the first mode for the different methods. The time was measured on a personal computer (PC) running two processors at $2.66 \mathrm{GHz}$ and 24.0 GB RAM. Parallel algorithms were not utilized except for the finite element in COMSOL and the Galerkin discretization. The convergence criterion was set to $10^{-10}$ in order to quantify the time taken by each method.

Table 3. Discretization and computation time for the different methods used in calculating the frequency of the first mode.

\begin{tabular}{lcc}
\hline \multicolumn{1}{c}{ Method } & $\begin{array}{c}\text { CPU Time (s) } \\
\text { Case (a) }\end{array}$ & $\begin{array}{c}\text { CPU Time (s) } \\
\text { Case (b) }\end{array}$ \\
\hline Galerkin Expansion* & 2.20 & 4.88 \\
Finite Element* & 22 & 33 \\
State Space & 2.20 & 2.65 \\
Power Series & 2.51 & 10.55 \\
Chebyshev tau method & 3.74 & 2.78 \\
\hline $\begin{array}{l}\text { (a) } \bar{\sigma}=50.62 \text { and } \alpha=99.71 ; \text { and }(\mathrm{b}) \\
\text { *Parallel algorithms were employed }\end{array}$ \\
\hline
\end{tabular}


We note from Table 3 that the state-space method takes less time than the other methods to compute the eigenvalue. Even though application of the modified Gram-Schmidt orthonormalization adds extra time to the process for high applied tensions and weights, the overall time taken is less than that taken by the other methods. When the weight and tension increase, the other methods require more descritization; that is, more polynomials in the Chebyshev approximation, more terms in the power-series method, a finer mesh in the finite element method, and more modes in the Galerkin procedure, resulting in an increase in the needed time. This can be observed by comparing Case (b) with Case (a) in Table 3.

\section{Parametric Investigation}

In this section, we investigate the effects of the parameters $\alpha$ (tension) and $\bar{\sigma}$ (weight) and the boundary conditions on the convergnece of the state-space scheme. The result obtained from the determinant in Eq. (20) is based on the two vectors in Eq. (18). Therefore, the 2-norm of the matrix consisting of the two final vectors is used as a measure to evaluate the numerical scheme. The tension ratio, defined as $T_{r}=\alpha / \bar{\sigma}$, is used to investigate the effects of the tension and weight on the state-space method. In the absence of orthonormalization, Fig. $2 \mathrm{a}, 2 \mathrm{~b}$, and $2 \mathrm{c}$ show that the increase in the tension imposes a limitation on the calculation of the first natural frequency for different end configuratios. We note that the numerical scheme breaks down when the norm reaches an order of $\left(10^{11}, 10^{12}\right)$. As the weight of the structure increases, the critical applied tension at which the numerical scheme breaks down (dashed line) decreases. Also, the

stiffness of the numerical scheme increases when the end configuration is changed from hingedhinged to fixed-fixed and fixed-hinged. This decreases the critical applied trension, as observed from comparing Fig. 2c and 2b with Fig. 2a. 

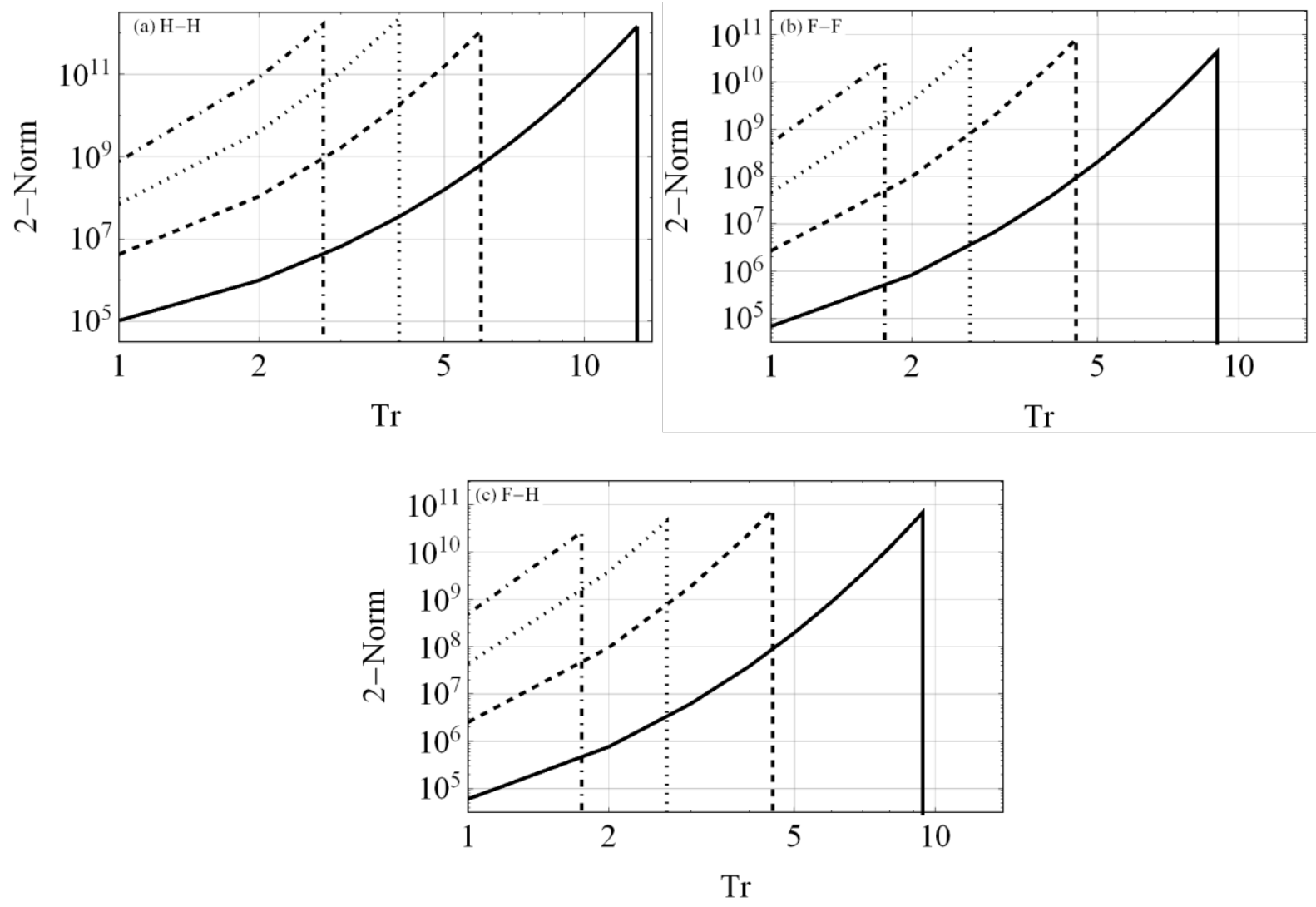

Figure 2. Variation of the 2-norm of the vectors from equation (18) with the tension ratio for various values of the weight: (a) hinged-hinged configuration, (b) fixed-fixed configuration, and (c) fixed-hinged configuration for the

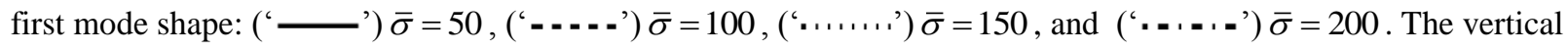
segment of each curve represents the critical tension in the absence of orthonormalization.

The break down of the numerical scheme is expected to occur for higher order modes.

Fig. 3 demonstrates how the numerical scheme breaks down in the absence of orthonormalization when the applied tension increases for a weight value of $\bar{\sigma}=100$. There is a critical tension below which an eigenvalue can be computed. Above the critical tension the numerical scheme fails due to the loss of linear independence of the computed vectors. The critical tension decreases as the mode number increases. 




Figure 3. Variation of the 2-norm of the vectors from equation (18) with the tension ratio for various modes of a

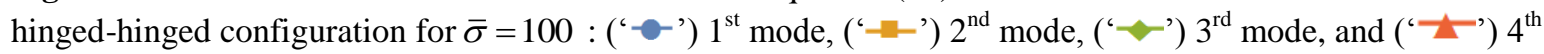
mode. The vertical segment of each curve represents the critical tension in the absence of orthonormalization.

It follows from Fig. 2 and 3 that it is necessary to introduce orthonormalization during the integration process for high tension values, high apparent weights, and high-order modes. This is because adding steps at the end of the integration does not influence how the integration process behaves.

Figure 4 shows the effect of introducing the orthonormalization process on computing the fundamental natural frequencies of the system. The vertical lines in Fig. 4 represent the critical tensions below which the natural frequencies can be computed using a number of orthonormalization steps. The zero step values are those obtained from Fig. 2a. Then, if the tension increases beyond that critical value, one needs to increase the orthonormalization steps in order to compute that natural frequency. It is also apparent from Fig. 4 that if the weight of the riser increases, more orthonormalization steps are needed to compute the natural frequency for high-tension values. 




Figure 4. Variation of the number of modified Gram-Schmidt orthonormalization steps with the tension ratio for various values of the weight of a hinged-hinged configuration for the first mode: ('- - ') $) \bar{\sigma}=50,\left({ }^{\prime}-{ }^{-}\right.$') $\bar{\sigma}=100$, $(‘ \sim$ ') $\bar{\sigma}=150$, and ('־`') $\bar{\sigma}=200$.

The effect of the number of orthonormalization steps on the calculation of the frequencies of the higher order modes is examined in Fig. 5. The number of orthonormalization steps needed to compute the frequencies increases for higher tensions and higher modes. Unlike Fig. 4, we note from Fig. 5 that the frequency of a higher order mode has little influence on the broadness of the tension value. Therefore, the greatest weight or highest mode can be established as a limit in order to apply the next orthonormalization step for all other modes or weights and compute the natural frequencies. Similarly, other configurations, such as hinged-fixed and fixed-fixed, exhibit similar characteristics when orthonormalization steps are introduced during the integration process.

We note that the state-space method can be extended to compute the buckling limits of risers. The buckling limit is obtained by setting $\omega=0$. Then, the aim is to find the applied axial force for which the steps from Eq. (14) to Eq. (20) are satisfied for a particular value of $\bar{\sigma}$. The numerical advantage in such cases is that orthonormalization is not needed except for higher order buckling modes. For example, the first buckling limit when $\bar{\sigma}=50$ for a clamped-hinged 
configuration is 51.0605 and the first buckling limit when $\bar{\sigma}=200$ is 121.6094 . These values are in good agreement with previously published results [8].

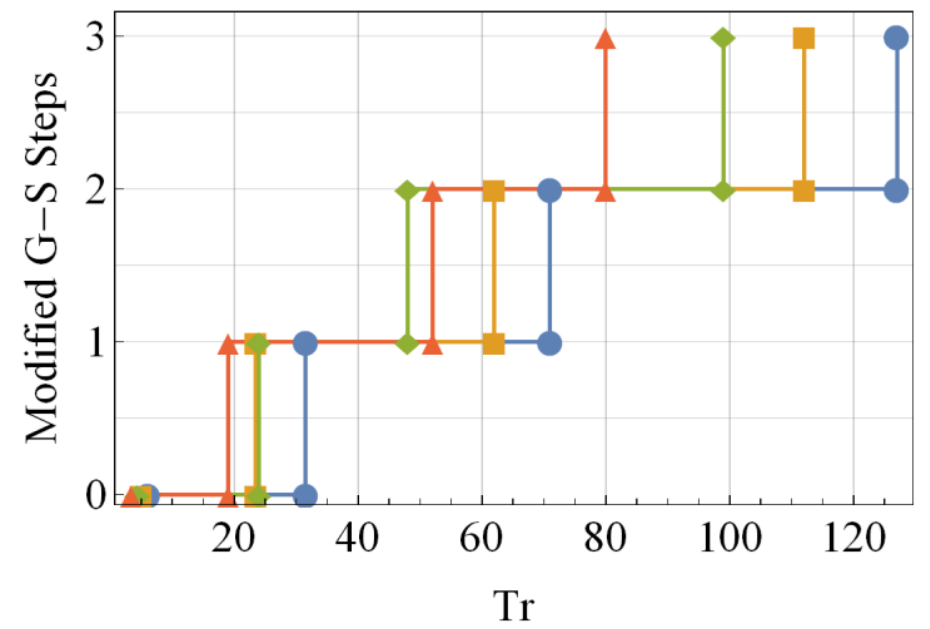

Figure 5. Variation of the number of modified Gram-Schmidt orthonormalization steps with the tension ratio for the modes of a hinged-hinged configuration for $\bar{\sigma}=100$ : ('-`) $1^{\text {st }}$ mode, (' $--^{-}$') $2^{\text {nd }}$ mode, (' $\neg$ ') $3^{\text {rd }}$ mode, and $\left(\right.$ ' - ') $4^{\text {th }}$ mode.

Next, we discuss the procedure for obtaining the mode shape. For large values of alpha and sigma, most boundary- value problem solvers are difficult to implement. One can use shooting of the boundary conditions techniques in conjunction with re-orthonormalization [33] to overcome the difficulties. Because the eigenvalues were found using the state-space method, we apply the shooting algorithm to find the roots for the initial-value problem. This is followed by introducing orthonormalization while numerically integrating the system. Therefore, in this manner the mode shape associated with each frequency is obtained. Depicted in Fig. 6 (a) are the lowest three mode shapes associated with case (b) in Table 3. In addition, we plot the lowest mode shapes for $\bar{\sigma}=500$ and $\alpha=-120$ in Fig. 6 (b). It is observed that a higher value of $\alpha$ shifts the maximum point of the mode shapes from the lower ends towards the middle. This causes the mode shape to behave like a near-symmetric mode shape in Fig. 6 (a), whereas the mode shapes in case Fig. 6 (b) exhibit more features of a Bessel mode shape depending on the values of alpha and sigma. 



Figure 6. Lowest three mode shapes of the vertical riser. (' $—$ ') $1^{\text {st }}$ mode shape, (' $\ldots . .$. ') $2^{\text {nd }}$ mode shape, and ('..... ') $3^{\text {rd }}$ mode shape for: (a) $\bar{\sigma}=200$ and $\alpha=600$ (b) $\bar{\sigma}=500$ and $\alpha=-120$.

It is worth to indicate that the presented state-space scheme has an advantage over the direct shooting scheme for the boundary condition. In the particular case of marine risers, the shooting technique will convert the linear eigenvalue problem into a nonlinear one, causing the stiffness of the system to increase and hence causing loss of stability of the shooting algorithm [34] . In addition, shooting requires a guess for the initial solution in the absence of the eigenvalue, which is computationally cumbersome.

We note that the presented state-space method is not applicable for solving marine riser systems with static deflection $[35,36]$. The deflection introduces a non-homogenous linear term from the geometric nonlinearity in which the numerical state of the end condition becomes nonzero. In this case, one can use other methods, such as the Galerkin procedure [11,35] and the finite element [37].

\section{CONCLUSIONS}

We used the state-space approach to compute the natural frequencies and buckling limits of vertical marine risers. The results are in good agreement with those obtained with other 
methods; however, the state-space approach is more numerically efficient than the other methods. In addition, we demonstrated the advantage of the modified Gram-Schmidt orthonormalization process. In the case of the frequencies of higher order modes or risers with large weight, orthonormalization prevents the numerical integration from diverging. In either case, the steps from an extreme case can be utilized to compute the natural frequencies for cases which pose less stringent orthonormalization requirement. Finally, the state-space method can be programed in packaged software for efficient computation of the linear dynamics of marine risers.

\section{ACKNOWLEDGMENTS}

This research was made possible through the fund and resources of the IT Research Computing at King Abdullah University of Science \& Technology (KAUST) in Thuwal, Saudi Arabia. Also, the first author acknowledges the support of Saudi Aramco.

\section{CONFLICT OF INTEREST}

The authors declare that they have no conflict of interest.

\section{APPENDIX A Convegence of the methods used to compute the riser eigenvalues}

We present convergence of the methods used to compute the first natural frequency presented in Table 2. For higher order modes and higher values of tension, more steps are needed in each method to achieve convergence.

1. Galerkin Method

In the Galerkin method, we use the mode shapes of a straight beam given by the boundary-value problem in [38]. Then, substituting the mode shapes and applying the orthogonality condition on 
Eq. (7) reduces it to a set of $n$ algebraic equations that need to be solved. The determinant of the matrix containing the coefficient of the algebraic equations gives the characteristic equation of the frequencies. Convergence of the frequency is shown in Fig. A.1.

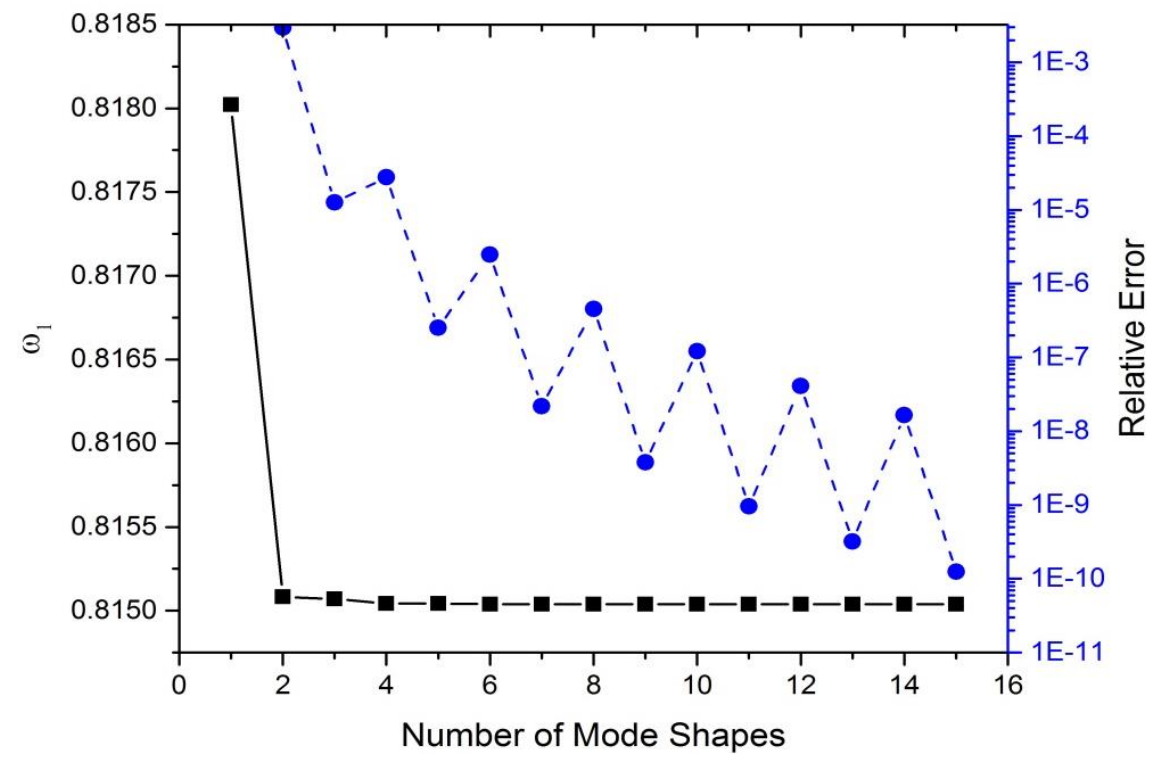

Figure A.1 Convergence of the first natural frequency of the riser versus the number of mode shapes: ('- - ') Relative Error and ('一匹-') Convergence of $\omega_{1}$.

2. Chebyshev tau method

In this method, the governing Eq. (7) is solved using a spectral decomposition in shifted Chebyshev polynomials [32]. The converged results are shown in Fig. A.2. 


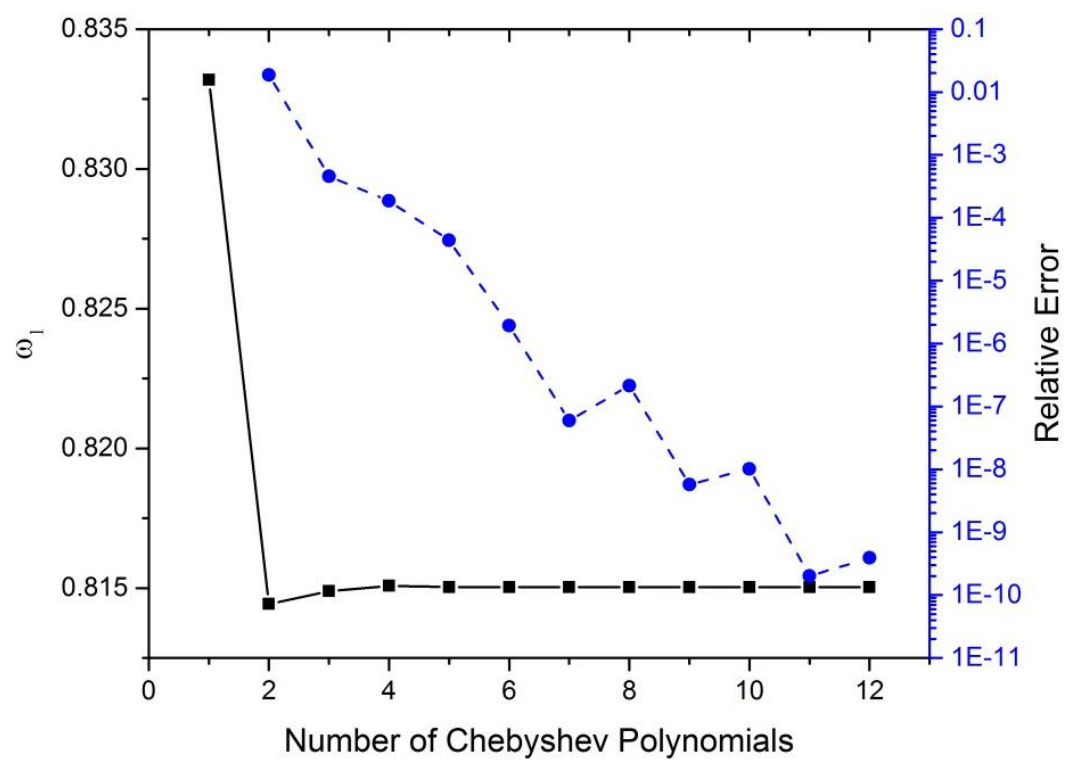

Figure A.2 Convergence of the first natural frequency of the riser equation versus the number of Chebyshev polynomials: ('- - -’) Relative Error and ('- - - ') Convergence of $\omega_{1}$.

It was shown in [14] that 320 Chebyshev polynomials are required to achieve convergence for the first natural frequency for tension dominated structures.

\section{Finite Element}

We use lagrange interpolation with quadratic shape functions in the FE procedure. The mesh is divided into three domains where an adaptive mesh is applied near each end. The solver is a built-in parallel computing algorithm given by the PARDISO solver [39]. A relative tolerance of $10^{-10}$ is used to compute the amplitude of the mode shape before the next mesh refining step. Convergence of the eigenvalue is shown in Fig. A.3. 


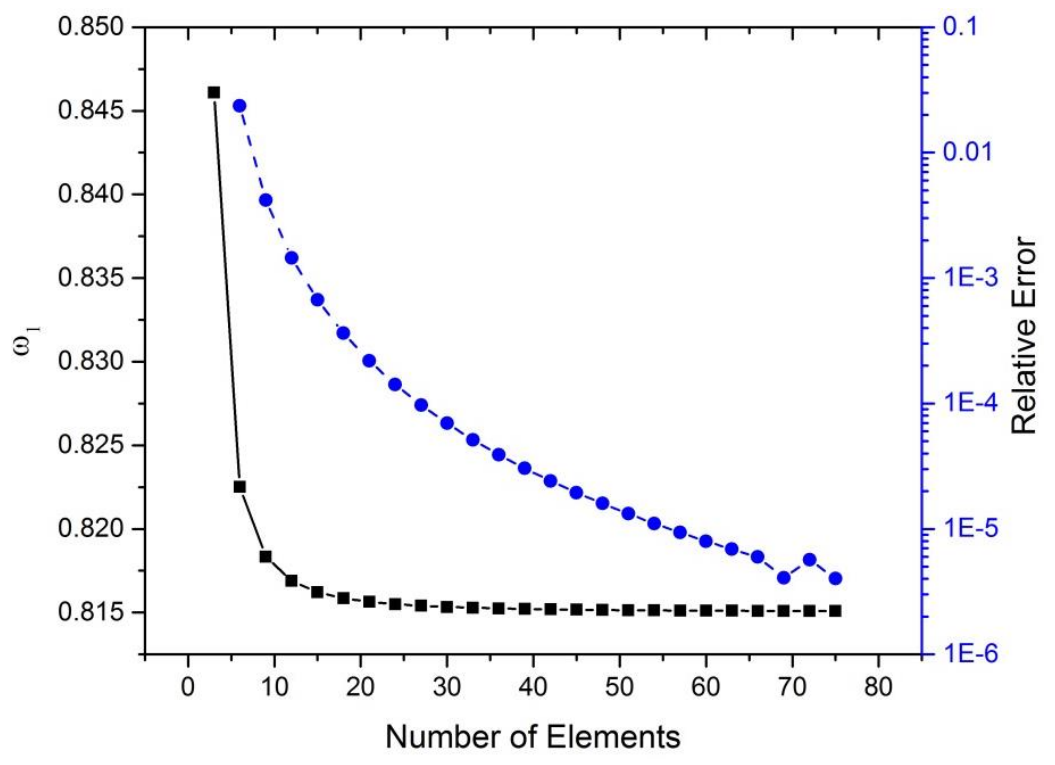

Figure A.3 Convergence of the first natural frequency of the riser equation from finite element solution versus number of elements used in the analysis: ('- - ') Relative Error and ('- - -') Convergence of $\omega_{1}$. 


\section{REFERENCES}

[1] L. Song, S. Fu, J. Cao, L. Ma, J. Wu, An investigation into the hydrodynamics of a flexible riser undergoing vortex-induced vibration, Journal of Fluids and Structures, 63 (2016) 325-350.

[2] C.E. Mazzilli, F. Rizza, T. Dias, Heave-imposed motion in vertical risers: a reduced-order modelling based on Bessel-like modes, Procedia IUTAM, 19 (2016) 136-143.

[3] H.L. Dai, A. Abdelkefi, L. Wang, Modeling and nonlinear dynamics of fluid-conveying risers under hybrid excitations, International Journal of Engineering Science, 81 (2014) 1-14.

[4] F. Clementi, L. Demeio, C.E.N. Mazzilli, S. Lenci, Nonlinear vibrations of non-uniform beams by the MTS asymptotic expansion method, Continuum Mechanics and Thermodynamics, 27 (2015) 703-717.

[5] W.-L. Chen, Q.-Q. Zhang, H. Li, H. Hu, An experimental investigation on vortex induced vibration of a flexible inclined cable under a shear flow, Journal of Fluids and Structures, 54 (2015) 297-311.

[6] G.R.S. Assi, N. Srinil, C.M. Freire, I. Korkischko, Experimental investigation of the flowinduced vibration of a curved cylinder in convex and concave configurations, Journal of Fluids and Structures, 44 (2014) 52-66.

[7] Y. Modarres-Sadeghi, F.S. Hover, M.S. Triantafyllou, Fatigue calculation of risers using a van der pol wake oscillator model with random parameters, American Society of Mechanical Engineers, New York, 2008.

[8] T. Huang, D.W. Dareing, Buckling and lateral vibration of drill pipe, Journal of Manufacturing Science and Engineering, 90 (1968) 613-619.

[9] D. Dareing, T. Huang, Natural frequencies of marine drilling risers, Journal of Petroleum Technology, 28 (1976) 813-818.

[10] M. Paidoussis, Dynamics of flexible slender cylinders in axial flow Part 1. Theory, Journal of Fluid Mechanics, 26 (1966) 717-736.

[11] C.L. Kirk, E.U. Etok, M.T. Cooper, Dynamic and static analysis of a marine riser, Applied Ocean Research, 1 (1979) 125-135.

[12] Y.C. Kim, M.S. Triantafyllou, The Nonlinear Dynamics of Long, Slender Cylinders, Journal of Energy Resources Technology, 106 (1984) 250-256.

[13] W.M. Laird, G. Fauconneau, Upper and lower bounds for the eigenvalues of vibrating beams with linearly varying axial load, in, DTIC Document, 1966.

[14] M.S. Triantafyllou, G.S. Triantafyllou, The paradox of the hanging string: An explanation using singular perturbations, Journal of Sound and Vibration, 148 (1991) 343-351. 
[15] M.C. Wu, J.Y.K. Lou, Effects of rigidity and internal flow on marine riser dynamics, Applied Ocean Research, 13 (1991) 235-244.

[16] I.K. Chatjigeorgiou, Solution of the boundary layer problems for calculating the natural modes of riser-type slender structures, Journal of Offshore Mechanics and Arctic Engineering, 130 (2007) 011003-011003.

[17] C.E.N.L. Mazzilli, S.;Demeio, L., Non-linear free vibrations of tensioned vertical risers, in: Proceedings of 8th european nonlinear dynamics conference - ENOC2014, Viena, Austria, 2014.

[18] A.H. Nayfeh, Introduction to Perturbation Techniques, Wiley, 2011.

[19] C. Sparks, Mechanical behavior of marine risers mode of influence of principal parameters, Journal of Energy Resources Technology, 102 (1980) 214-222.

[20] Y. Chen, Y.H. Chai, X. Li, J. Zhou, An extraction of the natural frequencies and mode shapes of marine risers by the method of differential transformation, Computers \& Structures, 87 (2009) 1384-1393.

[21] Y. Chen, J. Zhang, H. Zhang, X. Li, J. Zhou, Re-examination of natural frequencies of marine risers by variational iteration method, Ocean Engineering, 94 (2015) 132-139.

[22] K. Huang, H.-C. Chen, C.-R. Chen, Numerical scheme for riser motion calculation during 3D VIV simulation, Journal of Fluids and Structures, 27 (2011) 947-961.

[23] Y. Cheng, J.K. Vandiver, G. Moe, The Linear Vibration Analysis of Marine Risers Using the WKB-based Dynamic Stiffness Method, Journal of Sound and Vibration, 251 (2002) 750760.

[24] G. Moe, Y. Cheng, J.K. Vandiver, Riser Analysisby Means of Some Finite Element Approaches, Proceedings of ETCE/OMAE, (2002).

[25] M.R. Scott, H.A. Watts, Computational Solution of Linear Two-Point Boundary Value Problems via Orthonormalization, SIAM Journal on Numerical Analysis, 14 (1977) 40-70.

[26] J. Hadian, A.H. Nayfeh, Free vibration and buckling of shear-deformable cross-ply laminated plates using the state-space concept, Computers \& Structures, 48 (1993) 677-693.

[27] P.J. Holmes, Bifurcations to divergence and flutter in flow-induced oscillations: A finite dimensional analysis, Journal of Sound and Vibration, 53 (1977) 471-503.

[28] M.P. Paidoussis, Fluid-Structure Interactions, Academic Press, Oxford, 2014.

[29] Z. Aktas, H.J. Stetter, A classification and survey of numerical methods for boundary value problems in ordinary differential equations, International Journal for Numerical Methods in Engineering, 11 (1977) 771-796. 
[30] L. Fousse, G. Hanrot, V. Lef, \#232, vre, P. P, \#233, lissier, P. Zimmermann, MPFR: A multiple-precision binary floating-point library with correct rounding, ACM Trans. Math. Softw., 33 (2007) 13.

[31] Comsol, COMSOL Multiphysics: Version 4.3a, Comsol, 2012.

[32] S.A. Orszag, Accurate solution of the Orr-Sommerfeld stability equation, Journal of Fluid Mechanics, 50 (1971) 689-703.

[33] U.M. Ascher, R.M. Mattheij, R.D. Russell, Numerical solution of boundary value problems for ordinary differential equations, Siam, 1994.

[34] E. Cheney, D. Kincaid, Numerical Mathematics and Computing, Cengage Learning, 2012.

[35] F.K. Alfosail, A.H. Nayfeh, M.I. Younis, Natural frequencies and mode shapes of statically deformed inclined risers, International Journal of Non-Linear Mechanics, (2016).

[36] F.K. Alfosail, A.H. Nayfeh, M.I. Younis, An analytic solution of the static problem of inclined risers conveying fluid, Meccanica, (2016) 1-13.

[37] D. Meng, L. Chen, Nonlinear free vibrations and vortex-induced vibrations of fluidconveying steel catenary riser, Applied Ocean Research, 34 (2012) 52-67.

[38] L. Meirovitch, Fundamentals of Vibrations, McGraw-Hill, 2001.

[39] O. Schenk, K. Gärtner, W. Fichtner, A. Stricker, PARDISO: a high-performance serial and parallel sparse linear solver in semiconductor device simulation, Future Generation Computer Systems, 18 (2001) 69-78. 